\title{
DENSE ORBITS AND DOUBLE COSETS
}

\author{
JONATHAN BRUNDAN \\ Department of Pure Mathematics \\ 16, Mill Lane \\ Cambridge, CB2 1SB
}

\section{Introduction}

Throughout, $G$ denotes a connected reductive algebraic group defined over an algebraically closed field $k$ of characteristic $p \geq 0$. We are mainly interested here in positive characteristic.

In his article in these proceedings, Seitz considers the problem of finding closed subgroups $H$ and $P$ of $G$ for which there are finitely many $H, P$ double cosets in $G$; of particular interest is the case when $H$ is reductive and $P$ is parabolic. We will describe some results on the closely related (and often easier) problem of finding such subgroups $H$ and $P$ with a dense $H, P$-double coset in $G$.

We mention two special cases: first, let $\rho: H \rightarrow G=G L(V)$ be a rational representation of $H$ and let $P$ be the stabilizer in $G$ of a 1-space in $V$. Then, there is a dense $\rho H, P$-double coset in $G$ if and only if $H$ has a dense orbit on the set of 1-subspaces of $V$ - that is, $V$ is a prehomogeneous space for $H$. Irreducible prehomogeneous spaces have been classified in [26] $(p=0)$ and [5], [6] $(p>0)$.

Secondly, take $G$ to be arbitrary but let $P=B$ be a Borel subgroup of $G$. Then, subgroups $H$ of $G$ with a dense orbit on the flag variety $G / B$ - equivalently, a dense $H, B$-double coset - are called spherical subgroups, and the associated varieties $G / H$ are spherical varieties (see [3] for a survey of some geometric results). In characteristic 0 , all reductive spherical subgroups of $G$ have been classified, by Krämer [16] ( $G$ simple) and Brion [2], Mikityuk [20] ( $G$ reductive); there is at present no such classification in positive characteristic.

One aim of this article is to make precise a reduction modulo $p$ argument, assuming that the subgroup $H$ is 'defined over integers' in a suitable way. This argument depends on a representation-theoretic criterion for the 
existence of a dense $H, P$-double coset due to Kimel'fel'd and Vinberg, which we review in section 1.

The reduction modulo $p$ argument described in section 2 will sometimes allow us to deduce the existence of a dense $H, P$-double coset in characteristic $p$ from (often known) existence in characteristic 0. For this to work, we need the subgroup $H$ to satisfy an extra condition connected with good filtrations; we give some examples (and non-examples) of subgroups satisfying this extra condition in section 3. In particular, by a result of Donkin and Mathieu, all Levi subgroups satisfy the condition, so we obtain the classification of all spherical Levi subgroups immediately from Krämer's classification over $\mathbb{C}$. The problem of classifying more general spherical subgroups is discussed further in section 4.

We conclude the introduction with some remarks on the relationship between the existence of a dense $H, P$-double coset as discussed here and the existence of finitely many double cosets as in Seitz's problem. Of course, if there are finitely many $H, P$-double cosets in $G$ then there is a dense one, but the converse is false in general. For instance, take $H$ to be the unipotent radical of the opposite parabolic subgroup to $P$ : there is always a dense $H, P$-double coset in $G$, but there are finitely many double cosets if and only if the Dynkin diagram of $P$ is a union of connected components of the Dynkin diagram of $G$.

However, in the special case that $P=B$ is a Borel subgroup of $G$, the existence of a dense $H, B$-double coset in $G$ does imply that there are finitely many $H, B$-double cosets in $G$. This follows from the following remarkable result (with $X=G / H$ ), due to Brion [1] and Vinberg [29] in characteristic 0 and recently extended to positive characteristic by Knop [15, Corollary $2.6]$ :

Finiteness Theorem Suppose that $X$ is an irreducible $G$-variety on which $B$ has a dense orbit. Then, $B$ has finitely many orbits on $X$.

So, the reductive spherical subgroups give a family of examples in Seitz's finite orbit problem.

\section{Representation-theoretic interpretation}

In this section, we review results of Kimel'fel'd and Vinberg [14] giving representation-theoretic criteria for the existence of dense orbits. These results were originally proved only in characteristic 0 , but generalize quite easily to arbitrary characteristic, as is well known to several authors.

First, we set up notation that will be in place for the remainder of the article. Always, $G$ will denote a connected reductive algebraic group with fixed Borel subgroup $B$ containing a maximal torus $T$. Let $X(T)$ denote the character group of $T, W=N_{G}(T) / T$ denote the Weyl group, and choose 
a $W$-invariant inner product $\langle$,$\rangle on \mathbb{R} \otimes_{\mathbb{Z}} X(T)$. Let $\Phi \subset X(T)$ denote the root system of $G$. For $\alpha \in \Phi, \alpha^{\vee}$ denotes $\frac{2 \alpha}{\langle\alpha, \alpha\rangle}$. Let $\left\{\alpha_{i} \mid i \in I\right\}$ be a base for $\Phi$, chosen so that $B$ contains all negative root subgroups. For $\alpha \in \Phi$, let $U_{\alpha}$ denote the corresponding $T$-root subgroup of $G$ and for a subset $J$ of $I$, let $P_{J}$ denote the standard parabolic subgroup $\left\langle B, U_{\alpha_{j}} \mid j \in J\right\rangle$.

Let $X(T)^{+} \subset X(T)$ denote the dominant weights, i.e. the weights $\lambda$ satisfying $\left\langle\lambda, \alpha_{i}^{\vee}\right\rangle \geq 0$ for all $i \in I$. Let $\left\{\omega_{i} \mid i \in I\right\}$ denote the fundamental dominant weights, satisfying $\left\langle\omega_{i}, \alpha_{j}^{\vee}\right\rangle=\delta_{i j}$ for all $i, j \in I$. Our labelling of Dynkin diagrams is as in [11]. For $\lambda \in X(T)^{+}$let $\nabla_{G}(\lambda)$ denote the dual Weyl module $\operatorname{ind}_{B}^{G} \lambda$, and let $\Delta_{G}(\lambda)$ denote the corresponding Weyl module, which is the contravariant dual of $\nabla_{G}(\lambda)$.

Given an algebraic group $S$ and an irreducible $S$-variety $X$, we let $k(X)$ denote the function field of $X ; S$ acts on $k(X)$ by $(g . f)(x)=f\left(g^{-1} x\right)$ for $f \in k(X)$ defined at $x \in X$, and $g \in S$. We let $k(X)^{S}$ denote the $S$-fixed points in $k(X)$. We have the basic invariant-theoretic fact:

Lemma 1.1 (Rosenlicht, [25]) The transcendence degree of $k(X)^{S}$ over $k$ equals the minimum codimension of an $S$-orbit in $X$. In particular, $S$ has a dense orbit on $X$ if and only if $k(X)^{S}=k$.

Now we give the two results of Kimel'fel'd and Vinberg. The first is [14, Theorem 2]; we have included the proof since it is quite instructive.

Theorem 1.2 Let $X$ be an irreducible affine $G$-variety. Then, $B$ has a dense orbit on $X$ if and only if $\operatorname{Hom}_{G}\left(\Delta_{G}(\lambda), k[X]\right)$ is at most 1-dimensional for all $\lambda \in X(T)^{+}$.

Proof. For notational convenience, we work with the opposite Borel subgroup $B^{+}$to $B$. Suppose that $\operatorname{Hom}_{G}\left(\Delta_{G}(\lambda), k[X]\right)$ is at least 2-dimensional for some $\lambda$. Then, we can find linearly independent functions $f, g \in k[X]$ that are $B^{+}$-high weight vectors of weight $\lambda$. But then the quotient $f / g \in$ $k(X)$ is a non-constant $B^{+}$-invariant, so there is no dense $B^{+}$-orbit on $X$.

Conversely, suppose that there is no dense orbit, so that we can find a non-constant $B^{+}$-invariant function $\phi \in k(X)^{B^{+}}$by Lemma 1.1. As $X$ is affine, we can write $\phi=f / g$ for $f, g \in k[X]$. The $B^{+}$-submodule $V$ of $k[X]$ generated by $f$ is finite-dimensional, so we can find $b_{1}, \ldots, b_{n} \in B^{+}$ such that $V=\left\langle b_{1} . f, \ldots, b_{n} . f\right\rangle$. Now, by the Lie-Kolchin theorem, $B^{+}$fixes a 1-space in $V$, so there are scalars $c_{i} \in k$ such that $\tilde{f}:=\sum c_{i} b_{i} . f$ spans a $B^{+}$-stable 1-space, of weight $\lambda$ say. Let $\tilde{g}=\sum c_{i} b_{i} . g$.

Since $f / g$ is $B^{+}$-invariant, $f(b . g)=g(b . f)$ for all $b \in B^{+}$, so $g \tilde{f}=f \tilde{g}$, so $\tilde{f} / \tilde{g}=f / g$. This is $B^{+}$-invariant, so as $b . \tilde{f}=\lambda(b) \tilde{f}$ for all $b \in B^{+}$, the same is true for $\tilde{g}$. But $\phi$ is non-constant, so $\tilde{f}$ and $\tilde{g}$ are linearly independent $B^{+}$high weight vectors in $k[X]$ of high weight $\lambda$. Now the universal property of Weyl modules implies that $\operatorname{dim}_{H^{\prime}}\left(\Delta_{G}(\lambda), k[X]\right) \geq 2$. 
Recall that a subgroup $H$ of $G$ is spherical if it has a dense orbit on $G / B$. As a corollary to the theorem, we obtain a representation-theoretic criterion for reductive spherical subgroups:

Corollary 1.3 Let $H$ be a closed, connected reductive subgroup of $G$. Then, $H$ is spherical if and only if the fixed point space

$$
\nabla_{G}(\lambda)^{H}
$$

is at most 1-dimensional for all $\lambda \in X(T)^{+}$.

Proof. There is a dense $H$-orbit on $G / B$ if and only if there is a dense $B$-orbit on $G / H$. Now we note that the variety $G / H$ is an affine variety as $H$ is reductive, by [22], and apply Theorem 1.2 to deduce that there is a dense $B$-orbit on $G / H$ if and only if $\operatorname{Hom}_{G}\left(\Delta_{G}(\lambda), k[G / H]\right)$ is at most 1-dimensional for all $\lambda \in X(T)^{+}$. To complete the proof, the $G$-module $k[G / H]$ is precisely the induced module $\operatorname{ind}_{H}^{G} k$, so by Frobenius reciprocity $[13$, I.3.4 $], \operatorname{Hom}_{G}\left(\Delta_{G}(\lambda), k[G / H]\right) \cong \operatorname{Hom}_{H}\left(\Delta_{G}(\lambda), k\right)$. The result follows on dualizing.

Kimel'fel'd and Vinberg also prove a second result [14, Theorem 1], which gives a similar criterion for the existence of a dense $H, P$-double coset in $G$, for any closed subgroup $H$ and any parabolic $P$. A proof in arbitrary characteristic can be found in [4, Theorem 3.5]; the idea is similar to the proof of Theorem 1.2, this time exploiting the fact that if $G$ is a semisimple, simply-connected algebraic group, then $k[G]$ is a unique factorization domain [21].

Theorem 1.4 Let $H$ be a closed, connected subgroup of $G$. Let $P=P_{J}$ be a parabolic subgroup of $G$. There is a dense $H$-orbit on $G / P$ if and only if the fixed point space

$$
\left(\nabla_{G}(\lambda) \otimes \mu\right)^{H}
$$

is at most 1-dimensional for all 1-dimensional $H$-modules $\mu$ and all $\lambda \in$ $X(T)^{+}$such that $\left\langle\lambda, \alpha_{j}^{\vee}\right\rangle=0$ for all $j \in J$.

Corollary 1.3 and Theorem 1.4 are particularly useful in proving the non-existence of a dense double coset. For example, it is known that the Levi subgroup $H=G L_{n}(k) G L_{m}(k)<G L_{n+m}(k)=G$ is a spherical subgroup. In fact, providing $n \neq m$, the derived subgroup $H^{\prime}=S L_{n}(k) S L_{m}(k)$ is also spherical, but this is false if $n=m$ since then $H^{\prime}$ fixes a 2-dimensional subspace in the dual Weyl module $\wedge^{n} V$, where $V$ is the natural $G$-module.

On the other hand, the existence of dense double cosets can often be proved directly. I am grateful to R. Guralnick for allowing me to include his arguments in the following examples which we will refer to later: 
Lemma 1.5 (Guralnick) The following are spherical in all characteristics:

(i) $S O_{n}(k)<S L_{n}(k)$ for $n \geq 2$;

(ii) $S O_{n}(k) S O_{m}(k)<S O_{n+m}(k)$ for $n, m \geq 1$.

Proof. Let $V$ be a vector space endowed with a quadratic form $q$. We say that $W \leq V$ is a non-singular subspace if either the restriction to $W$ of the bilinear form coming from $q$ is non-degenerate, or $W$ is odd-dimensional in characteristic 2 and the bilinear form on $W$ has a 1-dimensional radical $R$ on which $q$ is non-zero.

(i) Let $F$ be a maximal flag for $S L_{n}(k)$ so that each subspace is nonsingular. Let $B$ be the Borel subgroup stabilizing this flag. We claim that $S O_{n}(k) \cap B$ is finite, which suffices to show that $S O_{n}(k)$ is spherical by dimension. Consider the 2-dimensional subspace $V_{2}$ in the flag; $B$ induces a subgroup of $\mathrm{O}_{2}$ on this subspace, acting as \pm 1 on a non-singular vector. This is a finite subgroup of $O_{2}$. Now, $B \cap S O_{n}(k)$ also preserves $V_{2}{ }^{\perp}$, so by induction $B \cap S O_{n}(k)$ is finite as desired.

(ii) Use induction on $n$. If either $n=1$ or $m=1$, we are done by [4, Theorem A]. So suppose $n, m \geq 2$. Let $V$ be the natural Weyl module for $G=S O_{n+m}(k)$, with quadratic form $q$. Suppose $H=S O_{n}(k) S O_{m}(k)$ is the stabilizer in $V$ of a non-singular $n$-dimensional subspace $W<V$. Pick a totally singular line $R$ such that $R^{\perp} \cap W$ is a non-singular subspace of $W$ of dimension $n-1$. Let $P$ be the parabolic subgroup of $G$ fixing $R$. Now, $S=P \cap H$ stabilizes $R^{\perp} \cap W, R$ and $W$, so Witt's theorem easily implies that $S \cong S O_{n-1}(k) S O_{m-1}(k)$. Given this, by dimensions we see that $H P$ is dense in $G$. Now let $L$ be a Levi subgroup of $P$ containing $S$. By induction, there is a Borel subgroup $B_{S}<S$ such that $S B_{S}$ is dense in $L$. So if $V$ is the unipotent radical of $P, B=B_{S} V$ is a Borel subgroup of $G$ and $S B$ is dense in $P$. So, $H B=H S B$ is dense in $H P$, which is dense in $G$.

\section{Integral embeddings}

We now consider reduction modulo $p$. We begin by recalling some basic facts about group schemes; we refer the reader to [13, I.2, II.1] for more details of these matters.

Let $R$ be a commutative ring and $\underline{S}$ be an $R$-group scheme (as in [13, I.2.1]); so $\underline{S}$ is the group scheme associated to a commutative Hopf algebra over $R$, namely its coordinate ring $R[\underline{S}]$. We say $\underline{S}$ is an algebraic $R$-group if $R[\underline{S}]$ is finitely presented, reduced if $R[\underline{S}]$ is reduced, and flat if $R[\underline{S}]$ is. Given an $R$-algebra $K$ and an $R$-module $M, M_{K}$ denotes $M \otimes_{R} K$; if $M$ is an $\underline{S}$-module (equivalently, an $R[\underline{S}]$-comodule) then $M_{K}$ is an $\underline{S}_{K}$-module. If $K$ is algebraically closed and $\underline{S}_{K}$ is a reduced algebraic $K$-group, we may regard it simply as a linear algebraic group over $K$, written $S_{K}$. We recall the universal coefficient theorem: 
Proposition 2.1 ([13, I.4.18]) Suppose that $R$ is a Dedekind ring and that $\underline{S}$ is a flat $R$-group scheme. Let $K$ be an $R$-algebra. Then, for any torsion free $\underline{S}$-module $M$ and any $i \in \mathbb{N}$, there is an exact sequence:

$$
0 \longrightarrow H^{i}(\underline{S}, M)_{K} \longrightarrow H^{i}\left(\underline{S}_{K}, M_{K}\right) \longrightarrow \operatorname{Tor}_{1}^{R}\left(H^{i+1}(\underline{S}, M), K\right) \longrightarrow 0 .
$$

Given our fixed connected reductive algebraic group $G$ over $k$, there is a split, reductive algebraic $\mathbb{Z}$-group $G$ such that $G_{k} \cong G$. For any $\operatorname{ring} R$, we obtain the scheme $\underline{G}_{R}$ on base change; note that $R\left[\underline{G}_{R}\right]$ is $R$-free, so $\underline{G}_{R}$ is flat.

Fix now a pair $(G, H)$ of connected reductive algebraic groups over $k$, where $H$ is a closed subgroup of $G$, and let $\underline{G}, \underline{H}$ denote corresponding split schemes over $\mathbb{Z}$. We say that $(G, H)$ is an integral embedding (defined over $R$ ) if there is an embedding $i: \underline{H}_{R} \rightarrow \underline{G}_{R}$ and an isomorphism $\theta: G_{k} \rightarrow G$ such that $\theta \circ i_{k}: H_{k} \rightarrow G$ is an isomorphism between $H_{k}$ and $H$, for some Dedekind ring $R$ with field of fractions of characteristic 0 for which $k$ is an $R$-algebra. Given any $R$-algebra $K$ that is an algebraically closed field, we let $\left(G_{K}, H_{K}\right)$ be the corresponding embedding over $K$, and we identify $\left(G_{k}, H_{k}\right)$ with $(G, H)$.

The following give examples of integral embeddings:

(1) any semisimple subgroup $H$ of $G=S L(V)$, where $H$ and the $H$ module $V$ arise via the Chevalley construction;

(2) any subgroup corresponding to a closed subsystem of the root system of $G$;

(3) centralizers of graph automorphisms of $G$;

(4) $S O_{n}(k) S O_{m}(k)<S O_{n+m}(k)$.

In fact, all these cases are integral embeddings defined over $\mathbb{Z}$, arising from embeddings of the Kostant $\mathbb{Z}$-forms $U(\mathbf{h})_{\mathbb{Z}} \rightarrow U(\mathbf{g})_{\mathbb{Z}}$ of the corresponding semisimple Lie algebras over $\mathbb{C}$. For more general integral embeddings, $R$ may need to be a ring of integers in an algebraic number field.

Now we obtain the following statement on reduction modulo $p$ :

Theorem 2.2 Suppose that $H$ is a closed, connected reductive subgroup of $G$ such that $(G, H)$ is an integral embedding defined over $R$. Let $K$ be the algebraic closure of the field of fractions of $R$ (of characteristic 0 ). Let $P$ be a standard parabolic subgroup of $G$ and let $P_{K}$ be the corresponding parabolic subgroup of $G_{K}$.

(i) If there is no dense $H_{K}, P_{K}$-double coset in $G_{K}$, there is no dense $H, P$-double coset in $G$.

(ii) If there is a dense $H_{K}, P_{K}$-double coset in $G_{K}$ and $H^{1}\left(H, \nabla_{G}(\lambda) \otimes\right.$ $\mu)=0$ for all $\lambda, \mu$ as in Theorem 1.4, then there is a dense H,P-double coset in $G$.

(iii) If $H_{K}$ is a spherical subgroup of $G_{K}$ and $H^{1}\left(H, \nabla_{G}(\lambda)\right)=0$ for all $\lambda \in X(T)^{+}$, then $H$ is spherical in $G$. 
Proof. By localizing if necessary, we may assume that $R$ is a DVR with irreducible element $\pi$ so that $k$ is an extension of the residue field $R / \pi R$. Let $\underline{H}_{R}<\underline{G}_{R}$ denote the corresponding schemes over $R$. We first claim:

For an $R$-free $\underline{H}_{R}$-module $M$ of finite rank, $\operatorname{dim}_{k}\left(M_{k}\right)^{H_{k}} \geq \operatorname{dim}_{K}\left(M_{K}\right)^{H_{K}}$. If $H^{1}\left(H_{k}, M_{k}\right)=0$ then equality holds.

To prove this, we may assume that $k$ actually equals the residue field $R / \pi R$. Note that $R$ is a PID so $M^{H_{R}}$ is $R$-free, of rank $d=\operatorname{dim}_{K}\left(M_{K}\right)^{H_{K}}$ as $K$ is flat over $R$. Proposition 2.1 with $i=0$ implies that $M^{H_{R}} \otimes k$ embeds injectively into $\left(M_{k}\right)^{H_{k}}$, so that $\operatorname{dim}_{k}\left(M_{k}\right)^{H_{k}} \geq d$. For the second part, let $A=H^{1}\left(\underline{H}_{R}, M\right)$ and suppose that $H^{1}\left(H_{k}, M_{k}\right)=0$. We just need to show that $\operatorname{Tor}_{1}^{R}(A, k)=0$, since then $M^{\underline{H}_{R}} \otimes k \cong\left(M_{k}\right)^{H_{k}}$. We know by our assumption and Proposition 2.1 with $i=1$ that $A \otimes k=0$. But $A \otimes k=0$ implies $A \otimes R / \pi R \cong A / \pi A=0$, so $\pi: A \rightarrow A$ is surjective. Since $A$ is a finitely generated $R$-module (which follows from $[8,1.7]$ ), the Nakayama lemma now implies that $\pi: A \rightarrow A$ is an isomorphism. Finally, $\operatorname{Tor}_{1}^{R}(A, R / \pi R)$ is the kernel of $\pi: A \rightarrow A$, so 0 .

We want to apply this to the module $\nabla_{G}(\lambda) \otimes \mu$ for $\lambda \in X(T)^{+}$and $\mu \in X(H)$. We need to note that this module is defined over $R$, that is, there is an $R$-free $\underline{H}_{R}$-module $M$ such that the $H_{k}$-module $M_{k}$ is isomorphic to $\nabla_{G}(\lambda) \otimes \mu$ (on identifying $H_{k}$ and $H$ ). This is clear for $\nabla_{G}(\lambda)$, since dual Weyl modules are even defined over $\mathbb{Z}$, and for the 1-dimensional $H$-module $\mu$ since $\underline{H}_{R}$ is a split reductive group.

The theorem now follows from the claim by Theorem 1.4 for (i) and (ii) and Corollary 1.3 for (iii).

We note that the conditions in parts (ii) and (iii) of the theorem are not necessary conditions for the dense orbit to be preserved on reduction modulo $p$ - compare Proposition 3.4 and Lemma 1.5 for examples.

\section{Good pairs}

We now consider how to verify the cohomological condition in Theorem 2.2. In fact, we use an even stronger condition to do with good filtrations which turns out to be easier to verify.

Recall that a $G$-module $V$ has a good filtration if it has an ascending filtration $0=V_{0}<V_{1}<V_{2}<\cdots$ with $V=\bigcup_{i \geq 0} V_{i}$ and each $V_{i} / V_{i-1}$ isomorphic to some $\nabla_{G}(\lambda)$ with $\lambda \in X(T)^{+}$. In that case, the dimension of $\operatorname{Hom}_{G}\left(\Delta_{G}(\lambda), V\right)$ simply computes the number of factors in any such filtration that are isomorphic to $\nabla_{G}(\lambda)$, by [13, II.4.16(a)]. The dual of a good filtration is a Weyl filtration.

There is also a cohomological criterion [13, II.4.16(b)]: $V$ has a good filtration if $H^{1}\left(G, V \otimes \nabla_{G}(\lambda)\right)=0$ for all $\lambda \in X(T)^{+}$. In particular, this 
cohomological criterion implies that if $V_{1}<V_{2}$ are $G$-modules with good filtrations, then $V_{2} / V_{1}$ also has a good filtration ([13, II.4.17]).

We say that $(G, H)$ is a good pair if $H$ is a closed, connected reductive subgroup of $G$ such that the restriction to $H$ of every $G$-module $V$ with a good filtration has a good filtration as an $H$-module. We say that $(G, H)$ is a good integral embedding if it is both a good pair and an integral embedding. By the cohomological criterion for good filtrations, if $(G, H)$ is a good integral embedding, then it satisfies the condition $H^{1}\left(H, \nabla_{G}(\lambda) \otimes \mu\right)=0$ in Theorem 2.2 (ii), for all $\lambda, \mu$.

We record the important theorem due to Donkin [7] (in almost all cases) and Mathieu [19] (in general):

Theorem 3.1 (i) If $V_{1}, V_{2}$ are $G$-modules with good filtrations, then $V_{1} \otimes V_{2}$ has a good filtration.

(ii) For any Levi subgroup $L$ of $G$, the pair $(G, L)$ is a good pair.

In particular, the theorem implies that the pair $(G, L)$ for any Levi subgroup of $G$ is a good integral embedding. To apply Theorem 2.2 to more general subgroups than Levi subgroups, we need some further examples of good integral embeddings. To construct these, the following basic facts will be useful:

Lemma 3.2 (i) If $(G, H)$ and $(H, L)$ are good pairs, so is $(G, L)$.

(ii) $(G, H)$ is a good pair if and only if $\left(G, H^{\prime}\right)$ is a good pair.

(iii) If $\left(G_{1}, H_{1}\right)$ and $\left(G_{2}, H_{2}\right)$ are good pairs, so is $\left(G_{1} \times G_{2}, H_{1} \times H_{2}\right)$.

(iv) For $G$ semisimple and simply-connected, $(G, H)$ is a good pair if and only if $\nabla_{G}\left(\omega_{i}\right) \downarrow_{H}$ has a good filtration for all $i \in I$.

(v) If $V$ is a finite-dimensional $G$-module with a Weyl filtration, and $v$ is a non-zero vector of maximal weight $\lambda$, then the $G$-submodule $W$ of $V$ generated by $v$ is isomorphic to $\Delta_{G}(\lambda)$, and $V / W$ has a Weyl filtration.

Proof. Part (i) is obvious and (ii) follows from [7, Proposition 3.2.7 (ii)]. For (iii), argue as in [7, Proposition 3.4.6] and for (iv) use [7, Proposition 3.5.4] combined with Theorem 3.1 (i). Finally, (v) is a result of Jantzen; see $[7,11.5 .1]$.

Proposition 3.3 The (simply-connected covers of the) following pairs are good integral embeddings:

(i) $(G, H)=\left(S L_{2 n}(k), S p_{2 n}(k)\right)(n>1)$;

(ii) $(G, H)=\left(S p_{2 n+2 m}(k), S p_{2 n}(k) S p_{2 m}(k)\right)(n, m \geq 1)$;

(iii) $(G, H)=\left(S L_{n}(k), S O_{n}(k)\right)(n \geq 1)$ if $p \neq 2$;

(iv) $(G, H)=\left(S O_{n+m}(k), S O_{n}(k) S O_{m}(k)\right)(n, m \geq 1)$ if either $p \neq 2$

or one of $n$ or $m$ is at most 2 ;

(v) $(G, H)=\left(S_{8}(k), \operatorname{Spin}_{7}(k)\right)$;

(vi) $(G, H)=\left(D_{4}, G_{2}\right)$ or $\left(B_{3}, G_{2}\right)$; 
(vii) $(G, H)=\left(G_{2}, A_{2}\right)$ or $\left(G_{2}, A_{1} \tilde{A}_{1}\right)$.

A proof is given in the appendix. We clarify some of the embeddings: the embedding of $\mathrm{Spin}_{7}$ in $\mathrm{SO}_{8}$ is the simply-connected group $\mathrm{B}_{3}$ embedded via a spin representation. In (vi), $G_{2}$ is the centralizer of a triality in a simply connected $D_{4}$, and lies in a subgroup of $D_{4}$ of type $B_{3}$. Finally, in the maximal rank subgroup $A_{1} \tilde{A}_{1}<G_{2}$ in (vii), $A_{1}$ denotes the long root $S L_{2}$, and $\tilde{A}_{1}$ denotes the short root one.

We conclude the section with a negative result showing that the restrictions in Proposition 3.3 (iii) and (iv) are necessary. This is interesting because it gives examples of maximal rank subgroups (coming from a closed subsystem of the root system) that are not good, in types $B$ or $D$. On the other hand, all such subgroups yield good pairs in all characteristics in types $A, C$ or $G$; I do not know about $E$ and $F$.

Proposition 3.4 Let $p=2$. Then the following are not good pairs:

(i) $(G, H)=\left(S L_{n}(k), S O_{n}(k)\right)(n \geq 3)$;

(ii) $(G, H)=\left(S O_{n+m}(k), S O_{n}(k) S O_{m}(k)\right)(n, m>2)$.

Proof. For (i), see the remark on [7, p. 75]. Now consider $(G, H)=$ $\left(S O_{n+m}(k), S O_{n}(k) S O_{m}(k)\right)$. If $n, m$ are both odd, let $V$ be the natural $G$ module. If $V \downarrow_{H}$ has a good filtration, it contains a dual Weyl module for $H$ as a submodule, necessarily one of $\nabla_{S O_{n}}\left(\omega_{1}\right)$ or $\nabla_{S O_{m}}\left(\omega_{1}\right)$ by character considerations; but this contradicts the fact that the socle of $V \downarrow_{H}$ is 1dimensional. It remains to consider the case where one of $n, m$ is even; here we will only sketch the argument.

Let $G$ be simply connected of type $B_{s+t}$ or $D_{s+t}$ and let $H$ be the maximal rank subgroup $B_{s} D_{t}(s \geq 1, t>1)$ or $D_{s} D_{t}(s, t>1)$ respectively. Let $\mathbf{g}, \mathbf{h}$ be the corresponding Lie algebras over $\mathbb{C}$ and $U(\mathbf{g})_{\mathbb{Z}}, U(\mathbf{h})_{\mathbb{Z}}$ be their Kostant $\mathbb{Z}$-forms coming from the choice of Chevalley basis made in $[12$, p. 38]. This choice of basis also fixes a triangular decomposition $U(\mathbf{g})_{\mathbb{Z}}=U(\mathbf{g})_{\mathbb{Z}}^{-} U(\mathbf{g})_{\mathbb{Z}}^{0} U(\mathbf{g})_{\mathbb{Z}}^{+}$. We fix the standard basis $e_{ \pm 1}, \ldots, e_{ \pm(s+t)}$, together with $e_{0}$ in type $B$, for the natural g-module $V$, again as in [12] or $\left[4\right.$, Section 2]. The $\mathbb{Z}$-span $V_{\mathbb{Z}}$ of this basis is precisely the $\mathbb{Z}$-form $U(\mathbf{g})_{\mathbb{Z}}^{-} e_{1}$ for $V$, and $U(\mathbf{h})_{\mathbb{Z}}$ is a subalgebra of $U(\mathbf{g})_{\mathbb{Z}}$ stabilizing the decomposition $V_{\mathbb{Z}}=$ $\left\langle e_{-s}, \ldots, e_{s}\right\rangle \oplus\left\langle e_{ \pm(s+1)}, \ldots, e_{ \pm(s+t)}\right\rangle$. The chosen triangular decomposition of $U(\mathbf{g})_{\mathbb{Z}}$ determines a corresponding decomposition of $U(\mathbf{h})_{\mathbb{Z}}$.

Now, the irreducible g-module $V\left(\omega_{2}\right)$ is precisely $\wedge^{2} V$; let $V\left(\omega_{2}\right)_{\mathbb{Z}}$ denote the $\mathbb{Z}$-form $U(\mathbf{g})_{\mathbb{Z}}^{-} \cdot e_{1} \wedge e_{2}$ in $\wedge^{2} V$. By [12, pp. 38-44], the following vectors give a basis for $V\left(\omega_{2}\right)_{\mathbb{Z}}$ over $\mathbb{Z}$ :

$\left\{e_{ \pm i} \wedge e_{ \pm j}\right\}_{1 \leq i<j \leq s+t} \cup\left\{e_{i} \wedge e_{-i}+e_{i+1} \wedge e_{-(i+1)}\right\}_{1 \leq i<s+t} \cup\left\{2 e_{s+t} \wedge e_{-(s+t)}\right\}$

together with $\left\{e_{ \pm i} \wedge e_{0}\right\}_{1 \leq i \leq s+t}$ if we are in type $B$. 
Using this, a direct calculation shows that $V\left(\omega_{2}\right)_{\mathbb{Z}}$ is generated as a $U(\mathbf{h})_{\mathbb{Z}}^{-}$-module by $e_{1} \wedge e_{s+1}, e_{s+1} \wedge e_{s+2}, e_{1} \wedge e_{-1}+e_{s+1} \wedge e_{-(s+1)}, e_{1} \wedge e_{2}$ if $H \neq B_{1} D_{b}, e_{1} \wedge e_{0}$ if $H=B_{1} D_{b}$ and $e_{1} \wedge e_{-2}$ if $H=D_{2} D_{b}$, but by no proper subset of these vectors. However, the vector $2\left(e_{1} \wedge e_{-1}+e_{s+1} \wedge e_{-(s+1)}\right)$ lies in the $U(\mathbf{h})_{\mathbb{Z}}^{-}$-submodule generated by all these vectors excluding $e_{1} \wedge e_{-1}+$ $e_{s+1} \wedge e_{-(s+1)}$.

Now, tensoring up with $k$ or $\mathbb{C}$, we have constructed a minimal set of weight vectors generating $V\left(\omega_{2}\right)_{k}$ as a $U(\mathbf{h})_{k}^{-}$-module which contains one more vector than a minimal set of weight vectors generating $V\left(\omega_{2}\right)_{\mathbb{C}}$ as a $U(\mathbf{h})_{\mathbb{C}}^{-}$-module. But this implies that $V\left(\omega_{2}\right)_{k} \cong \Delta_{G}\left(\omega_{2}\right)$ does not have a Weyl filtration as an $H$-module, since in that case any such minimal set over $k$ has the same size as over $\mathbb{C}$ because of Lemma $3.2(\mathrm{v})$.

\section{Spherical subgroups}

Now we discuss one special case, namely the classification of reductive spherical subgroups. As we mentioned in the introduction, all reductive spherical subgroups of $G$ have been classified over $\mathbb{C}$ in $[2,16,20]$. In positive characteristic, many examples of spherical subgroups are known thanks to a result of Springer [27, Lemma 4.1] which shows that, providing $p \neq 2$, any centralizer $K$ of an involution of $G$ is spherical. Such subgroups $K$, or rather the associated symmetric varieties $G / K$, have been studied by many authors (see e.g. [10, 17, 23]).

In [16], Krämer classified all pairs $\left(G_{\mathbb{R}}, H_{\mathbb{R}}\right)$ of compact, connected Lie groups, with $G_{\mathbb{R}}$ simple and $H_{\mathbb{R}}<G_{\mathbb{R}}$, satisfying the analogous representation-theoretic condition to the one in Theorem 1.3. Equivalently by [14], Krämer's results classify all pairs $\left(G_{\mathbb{C}}, H_{\mathbb{C}}\right)$ of connected reductive algebraic groups over $\mathbb{C}$ with $G_{\mathbb{C}}$ simple such that $H_{\mathbb{C}}$ is a spherical subgroup of $G_{\mathbb{C}}$. We list all such pairs $\left(G_{\mathbb{C}}, H_{\mathbb{C}}\right)$ from Krämer's classification in table 1 . We note that the property that $H_{\mathbb{C}}$ is spherical in $G_{\mathbb{C}}$ is preserved by isogenies of $G_{\mathbb{C}}$, so we only list the embeddings for our favourite representative of the root datum of $G_{\mathbb{C}}$. In the left hand side of table 1, we have listed all pairs for which $H_{\mathbb{C}}$ is (the connected component of) a centralizer of an involution of $G_{\mathbb{C}}$, and the remaining cases are listed in the right hand side. In the table, we denote a 1-dimensional central torus of $H$ by $T_{1}$. Also note that the embeddings of $\operatorname{Spin}_{7}<S O_{9}$ and $\operatorname{Spin}_{7} S_{2}<S O_{10}$ are via $\operatorname{Spin}_{7}<\mathrm{SO}_{8}<\mathrm{SO}_{9}$ and $\operatorname{Spin}_{7} \mathrm{SO}_{2}<\mathrm{SO}_{8} \mathrm{SO}_{2}<\mathrm{SO}_{10}$ respectively.

All the embeddings in table 1 can be considered equally well over our field $k$ of arbitrary characteristic (the only ambiguity being $S O_{2 n+1}(k)<$ $S L_{2 n+1}(k)$ if $p=2$, where we mean either of the two classes of such a subgroup). There is at present no complete classification of reductive spherical subgroups in characteristic $p$. We give here two partial results. 
TABLE 1. Krämer's spherical subgroups

\begin{tabular}{llllll}
\hline$G_{\mathbb{C}}$ & $H_{\mathbb{C}}$ & & $G_{\mathbb{C}}$ & $H_{\mathbb{C}}$ & \\
\hline \hline$S L_{n+m}$ & $S L_{n} S L_{m} T_{1}$ & $n, m \geq 1$ & $S L_{n+m}$ & $S L_{n} S L_{m}$ & $n \neq m$ \\
$S L_{2 n}$ & $S p_{2 n}$ & $n \geq 2$ & $S L_{2 n+1}$ & $S p_{2 n} T_{1}$ & $n \geq 1$ \\
$S L_{n}$ & $S O_{n}$ & $n \geq 2$ & $S L_{2 n+1}$ & $S p_{2 n}$ & $n \geq 1$ \\
$S p_{2 n+2 m}$ & $S p_{2 n} S p_{2 m}$ & $n, m \geq 1$ & $S p_{2 n+2}$ & $S p_{2 n} T_{1}$ & $n \geq 1$ \\
$S p_{2 n}$ & $G L_{n}$ & $n \geq 1$ & $S O_{2 n+1}$ & $G L_{n}$ & $n \geq 2$ \\
$S O_{n+m}$ & $S O_{n} S O_{m}$ & $n, m \geq 1$ & $S O_{4 n+2}$ & $S L_{2 n+1}$ & $n \geq 1$ \\
$S O_{2 n}$ & $G L_{n}$ & $n \geq 2$ & $S O_{7}$ & $G_{2}$ & \\
& & & $S O_{8}$ & $G_{2}$ & \\
& & & $S O_{8}$ & $S p_{2} \otimes S p_{4}$ & \\
& & & $S O_{8}$ & $S p i n_{7}$ & \\
& & & $S O_{9}$ & $S p i n_{7}$ & \\
$G_{2}$ & $A_{1} A_{1}$ & & $S O_{10}$ & $S p i n_{7} S O_{2}$ & \\
$E_{6}$ & $T_{1} D_{5}$ & & $G_{2}$ & $A_{2}$ & \\
$E_{7}$ & $T_{1} E_{6}$ & & $E_{6}$ & $D_{5}$ & \\
\hline$F_{4}$ & $B_{4}$ & & & & \\
$F_{4}$ & $A_{1} C_{3}$ & & & & \\
$E_{6}$ & $F_{4}$ & & & & \\
$E_{6}$ & $C_{4}$ & & & & \\
$E_{6}$ & $A_{1} A_{5}$ & & & & \\
$E_{7}$ & $A_{7}$ & & & & \\
$E_{7}$ & $A_{1} D_{6}$ & & & & \\
$E_{8}$ & $D_{8}$ & & & & \\
$E_{8}$ & $A_{1} E_{7}$ & & & & \\
\hline & & & & & \\
\hline
\end{tabular}

First, we have the classification of spherical Levi subgroups in arbitrary characteristic, which is immediate by Krämer's result and Theorem 2.2:

Theorem 4.1 Let $L$ be a Levi subgroup of $G$. Let $G^{\prime}=\prod_{i=1}^{r} G_{i}$ as a commuting product of simple factors and set $L_{i}:=L \cap G_{i}$. Then, $L$ is spherical in $G$ if and only if, for each $i$, either $G_{i}=L_{i}$ or $\left(G_{i}, L_{i}^{\prime}\right)$ is one of $\left(A_{n}, A_{m} A_{n-m-1}\right),\left(B_{n}, B_{n-1}\right),\left(B_{n}, A_{n-1}\right),\left(C_{n}, C_{n-1}\right),\left(C_{n}, A_{n-1}\right)$, $\left(D_{n}, D_{n-1}\right),\left(D_{n}, A_{n-1}\right),\left(E_{6}, D_{5}\right)$ or $\left(E_{7}, E_{6}\right)$.

This result also gives a classification of all parabolic subgroups $P$ of $G$ for which a Borel subgroup of a Levi factor of $P$ has a dense orbit (equivalently, finitely many orbits) on the unipotent radical of $P$, acting by conjugation. This follows because of the following observation (see $[2$, Proposition 1.1] for a more general result):

Lemma 4.2 Let $P$ be a parabolic subgroup of $G$ with Levi decomposition $P=L V$, where $V=R_{u}(P)$, and let $B_{L}$ be a Borel subgroup of $L$. There is a dense $L$-orbit on $G / B$ if and only if there is a dense $B_{L}$-orbit on $V$. 
Proof. We may assume that the Borel subgroup $B$ of $G$ is contained in $P$ and that $B_{L}=B \cap L$. Let $P^{+}=L V^{+}$be the opposite parabolic to $P$. Now, $L$ has a dense orbit on $G / B$ if and only if $L \times B$ has a dense orbit on $P^{+} B$, since $P^{+} B$ is dense in $G$. The map $P^{+} \times V \rightarrow P^{+} B$ given by multiplication is an isomorphism of varieties, so this is equivalent to $L \times B$ having a dense orbit on $P^{+} \times V$, where the action of $L$ is by left multiplication on $P^{+}$, and the action of an element $u b \in B\left(b \in B_{L}, u \in V\right)$ on $(p, v) \in P^{+} \times V$ is $u b$. $(p, v)=\left(p b^{-1}, b v b^{-1} u^{-1}\right)$. Since the orbits of $L \times B$ on $P^{+} \times V$ under this action are of the form $L p B_{L} \times V$ for $p \in P^{+}$, this is if and only if $L \times B_{L}$ has a dense orbit on $P^{+}$, i.e. $B_{L}$ has a dense orbit on $P^{+} / L$. Now the quotient map $V^{+} \rightarrow P^{+} / L$ is an isomorphism of varieties, so this is equivalent to $B_{L}$ having a dense orbit on $V^{+}$, and hence $V$.

In fact, for all of the pairs listed in Theorem 4.1 except for $\left(B_{n}, A_{n-1}\right)$ and $\left(C_{n}, C_{n-1}\right)$, the unipotent radical $V$ of $P$ is abelian. This abelian unipotent radical case has been studied by a number of authors: for instance, in [24], the number of $L$-orbits on $V^{+}$is shown to equal the number of $P, P$ double cosets in $G$. Also, Vavilov $[28, \S 4]$ has given a direct proof that there are finitely many $B_{L^{-}}$orbits on $V^{+}$here.

The proof of the next theorem has only recently been completed, thanks to work of Lawther [18]. Lawther's results show that there are finitely many double cosets for all the cases in the bottom half of table 1 , even in characteristic 2. Of course, in characteristic different from 2, these cases are centralizers of involutions, so finiteness follows from Springer's result. Lawther's approach in characteristic 2 involves bounding the number of double cosets for the corresponding finite groups of Lie type, using DeligneLusztig character theory.

Theorem 4.3 The entries in table 1 are spherical in all characteristics.

Proof. The cases $\left(S L_{n}, S O_{n}\right)$ and $\left(S O_{n+m}, S O_{n} S O_{m}\right)$ are verified in Lemma 1.5, and $\left(\mathrm{SO}_{8}, \mathrm{Sp}_{2} \otimes \mathrm{Sp}_{4}\right)$ follows from $\left(\mathrm{SO}_{8}, \mathrm{SO}_{3} \mathrm{SO}_{5}\right)$ on applying triality. The cases in the bottom half of table 1 follow from [27, Lemma $4.1](p \neq 2)$ or $[18](p=2)$. The remaining entries in table 1 are immediate from Theorem 2.2, Lemma 3.2, Theorem 3.1 (ii) and the list of good pairs in Proposition 3.3.

We conclude with a conjecture motivated by the article; there is some hope for a case-free proof of this (for part (i) it only remains to show that $\left(E_{6}, F_{4}\right)$ is a good pair, given Proposition 3.3).

Conjecture 4.4 Let $H$ be a closed reductive subgroup of $G$. If either

(i) $H$ is the centralizer of a graph automorphism of $G$; or

(ii) $H$ is the centralizer of an involution of $G$, then $(G, H)$ is a good pair. 


\section{A. Proof of Proposition 3.3}

In this appendix, we give the proof of Proposition 3.3. We will verify each case in the proposition in turn, using Lemma 3.2 (iv).

Part (i) is immediate from the following result of Donkin:

Lemma A.1 ([9, Appendix A $])$ Let $G=S p_{2 n}(k)$, with natural module $V$. Then for $1 \leq r \leq n, \wedge^{r} V$ has a filtration

$$
0=\left(\bigwedge^{r} V\right)_{0}<\cdots<\left(\bigwedge^{r} V\right)_{m}=\bigwedge^{r} V
$$

such that

$$
\left(\bigwedge^{r} V\right)_{i} /\left(\bigwedge^{r} V\right)_{i-1} \cong \begin{cases}\nabla_{G}\left(\omega_{2 i}\right) & r \text { even }, \\ \nabla_{G}\left(\omega_{2 i+1}\right) & r \text { odd }\end{cases}
$$

for all $0<i \leq m$, where $m=\left[\frac{r}{2}\right]$.

Consider the pair $(G, H)=\left(S p_{2 n+2 m}(k), S p_{2 n}(k) S p_{2 m}(k)\right)$. We have just shown that $\left(S L_{2 n}(k), S p_{2 n}(k)\right)$ is a good pair, so Lemma 3.2 (iii) implies that $\left(S L_{2 n}(k) S L_{2 m}(k), S p_{2 n}(k) S p_{2 m}(k)\right)$ is a good pair. Also, by Theorem 3.1 (ii) and Lemma 3.2 (ii), $\left(S L_{2 n+2 m}(k), S L_{2 n}(k) S L_{2 m}(k)\right)$ is a good pair. Combining, it follows that $\left(S L_{2 n+2 m}(k), S p_{2 n}(k) S p_{2 m}(k)\right)$ is a good pair. Now the conclusion follows from the next lemma:

Lemma A.2 Let $G=S L_{2 n}(k), H=S p_{2 n}(k)$. Suppose $L<H<G$ is a connected reductive algebraic group. Then $(H, L)$ is a good pair if and only if $(G, L)$ is a good pair.

Proof. The forward implication is immediate by Lemma 3.2 (i), so consider the converse. By Lemma 3.2 (iv), we need to check that $\nabla_{H}\left(\omega_{i}\right)$ has a good filtration as an $L$-module for each $1 \leq i \leq n$. For $i=1$, $V=\nabla_{H}\left(\omega_{1}\right) \cong \nabla_{G}\left(\omega_{1}\right) \downarrow_{H}$, so has a good filtration as an $L$-module as $(G, L)$ is a good pair. In general, $\nabla_{G}\left(\omega_{i}\right)=\bigwedge^{i} V$ has a good filtration as an $L$-module by assumption, and contains an $H$-submodule isomorphic to $\bigwedge^{i-2} V$ by Lemma A.1. This submodule has a good filtration as an $L$ module by assumption, hence so does the quotient. But this quotient is precisely $\nabla_{H}\left(\omega_{i}\right)$, completing the proof.

Next, we look at Proposition 3.3 (iii), (iv) and (v). If $p \neq 2$ and $G$ is the simply-connected cover of $S O_{n}(k)$, we note that the fundamental modules $\nabla_{G}\left(\omega_{i}\right)$ are all irreducible, by for instance [12, Satz I.13]. So, excluding the spin modules, these modules coincide with the exterior power $\bigwedge^{i} V$, where $V$ is the natural $G$-module. Now Proposition 3.3 (iii) and (iv) for $p \neq 2$ are obvious using Lemma 3.2 (iv). For $p=2$, we need to show $\left(S O_{n+m}(k), S O_{n}(k) S O_{m}(k)\right)$ is a good pair if $m \leq 2$. If $m=2$, this is just a Levi subgroup, so the result follows from Theorem 3.1. If $m=1$, this is proved in [4, Theorem D] (unfortunately though the proof 
in [4] for $D_{\ell}<B_{\ell}$ is not quite complete, though the result is correct). The case $\left(\mathrm{SO}_{8}(k), \mathrm{Spin}_{7}(k)\right)$ follows from $\left(\mathrm{SO}_{8}(k), \mathrm{SO}_{7}(k)\right)$ on applying triality (working in the simply-connected cover of $S_{8}(k)$ where triality is defined).

For the remaining two cases, we will use the fact that if $G$ is a simple, simply-connected group, then $\operatorname{Lie}(G)$ is a Weyl module. First, for Proposition 3.3 (vi), let $G$ be the simply connected group of type $D_{4}$ and $H=G_{2}$. We can view $H$ as $L \cap L^{\prime}$, where $L$ is a simply-connected group of type $B_{3}$ coming from the natural embedding $S_{7}(k)<S O_{8}(k)$, and $L^{\prime}$ is the image of $L$ under a triality. Consider the natural $G$-module $\Delta_{G}\left(\omega_{1}\right)$ of dimension 8. Since $\Delta_{G}\left(\omega_{1}\right) \downarrow_{L}$ contains the 7-dimensional module $\Delta_{L}\left(\omega_{1}\right)$ as a submodule, it has a Weyl filtration on restriction to $H$. Now applying triality yields that $\Delta_{G}\left(\omega_{3}\right)$ and $\Delta_{G}\left(\omega_{4}\right)$ also have Weyl filtrations as $H$-modules. So, it remains to show that $\Delta_{G}\left(\omega_{2}\right) \cong \operatorname{Lie}(G)$ has a Weyl filtration on restriction to $H$. A direct check using Chevalley bases shows that $\operatorname{Lie}(G)=\operatorname{Lie}(L)+\operatorname{Lie}\left(L^{\prime}\right)$, hence that $\operatorname{Lie}(L) \cap \operatorname{Lie}\left(L^{\prime}\right)=\operatorname{Lie}\left(L \cap L^{\prime}\right)=$ $\operatorname{Lie}(H)$ by dimension. Now we claim that $0<\operatorname{Lie}(H)<\operatorname{Lie}(L)<\operatorname{Lie}(G)$ is a Weyl filtration as an $H$-module. Certainly, $\operatorname{Lie}(H) \cong \Delta_{H}\left(\omega_{2}\right)$. Since $(G, L)$ is a good pair by (iv), Lemma $3.2(\mathrm{v})$ implies that $\operatorname{Lie}(G) / \operatorname{Lie}(L)$ is a Weyl module, necessarily $\Delta_{L}\left(\omega_{1}\right)$, which has a Weyl filtration as an $H$-module. The same argument shows that $\operatorname{Lie}(G) / \operatorname{Lie}\left(L^{\prime}\right)$ has a Weyl filtration as an $H$-module; but $\operatorname{Lie}(G) / \operatorname{Lie}\left(L^{\prime}\right)=\operatorname{Lie}(L)+\operatorname{Lie}\left(L^{\prime}\right) / \operatorname{Lie}\left(L^{\prime}\right) \cong$ $\operatorname{Lie}(L) / \operatorname{Lie}(L) \cap \operatorname{Lie}\left(L^{\prime}\right)=\operatorname{Lie}(L) / \operatorname{Lie}(H)$. These arguments also show that $(L, H)$ is a good pair.

Now let $G=G_{2}$. To prove (vii), there are two $G$-modules to check for Weyl filtrations: the 7-dimensional module $\Delta_{G}\left(\omega_{2}\right)$ and the 14-dimensional Lie algebra. For $H=A_{2}, \Delta_{G}\left(\omega_{2}\right)$ is semisimple as an $H$-module (factors of dimension $1,3,3)$ and the module $\operatorname{Lie}(G)$ contains $\operatorname{Lie}(H)=\Delta_{H}\left(\omega_{1}+\omega_{2}\right)$ as a submodule, and the quotient is semisimple (factors of dimension 3,3 ); so both have Weyl filtrations here. For $H=A_{1} \tilde{A}_{1}$, we note that $\Delta_{G}\left(\omega_{2}\right) \downarrow_{H}$ has the same character as $\Delta_{A_{1}}\left(\omega_{1}\right) \otimes \Delta_{\tilde{A}_{1}}\left(\omega_{1}\right) \oplus \Delta_{\tilde{A}_{1}}\left(2 \omega_{1}\right)$, so it is a direct sum of irreducible Weyl modules as an $A_{1}$-module, and has a Weyl filtration as an $\tilde{A}_{1}$-module by Theorem 3.1 ; this is enough to show (using Lemma 3.2 (v)) that it has a Weyl filtration as an $A_{1} \tilde{A}_{1}$-module. Also, by considering weights we see that the Lie algebra splits as a direct sum of $\operatorname{Lie}(H)$ together with a module with the same character as $\Delta_{A_{1}}\left(\omega_{1}\right) \otimes \Delta_{\tilde{A}_{1}}\left(3 \omega_{1}\right)$, so this summand is again a direct sum of irreducible Weyl modules as an $A_{1^{-}}$ module and has a Weyl filtration as an $\tilde{A}_{1}$-module by Theorem 3.1. Hence it has a Weyl filtration as an $A_{1} \tilde{A}_{1}$-module as desired.

This completes the proof of Proposition 3.3. 


\section{References}

1. Brion, M. (1986) Quelques propriétés des espaces homogènes sphériques, Man. Math. 55, 191-198.

2. Brion, M. (1987) Classification des espaces homogènes sphériques, Compositio Math. 63, 189-208.

3. Brion, M. (1994) Spherical varieties, Proceedings of the International Congress of Mathematicians, Zürich, pp. 753-760.

4. Brundan, J. (1997) Multiplicity-free subgroups of reductive algebraic groups, J. Algebra 188, 310-330.

5. Chen, Z. (1986) A classification of irreducible prehomogeneous vector spaces over an algebraically closed field of characteristic 2, I, Acta Math. Sinica 2, 168-177.

6. Chen, Z. (1988) A classification of irreducible prehomogeneous vector spaces over an algebraically closed field of characteristic p, II, Ann. Math. Ser. A 9, 10-22.

7. Donkin, S. (1985), Rational representations of algebraic groups: Tensor products and filtrations, Lecture Notes in Math. 1140, Springer-Verlag, New York.

8. Donkin, S. (1993) On tilting modules for algebraic groups, Math. Z. 212, 36-60.

9. Donkin, S. (1994) On tilting modules and invariants for algebraic groups, in V. Dlab and L.L. Scott (eds.), Finite dimensional algebras and related topics, Kluwer Academic Publishers, Dordrecht, pp. 59-77.

10. Helminck, A.G. (1996) Computing B-orbits on G/H, J. Symbolic Comput. 21, 169 209.

11. Humphreys, J.E. (1994) Introduction to Lie Algebras and Representation Theory, Springer-Verlag, New York, sixth edition.

12. Jantzen, J.C. (1973) Darstellungen halbeinfacher algebraischer Gruppen und zugeordnete kontravariante Formen, Bonner Math. Schriften No. 67, 1-124.

13. Jantzen, J.C. (1987) Representations of algebraic groups, Academic Press, Orlando.

14. Kimel'fel'd, B. and Vinberg, E.B. (1978) Homogeneous domains on flag manifolds and spherical subgroups of semisimple Lie groups, Funct. Anal. Appl. 12, 168-174.

15. Knop, F. (1995) On the set of orbits for a Borel subgroup, Comm. Math. Helv. 70, 285-309.

16. Krämer, M. (1979) Sphärische Untergruppen in kompakten zusammenhängenden Liegruppen, Compositio Math. 38, 129-153.

17. Lawther, R. (1995) Double cosets involving involutions in algebraic groups, Proc. London Math. Soc. 70, 115-145.

18. Lawther, R. (1998) Finiteness of double coset spaces, in preparation.

19. Mathieu, O. (1990) Filtrations of G-modules, Ann. Sci. Ecole Norm. Sup. 23, 625644 .

20. Mikityuk, V. (1987) On the integrability conditions of invariant Hamiltonian systems with homogeneous configuration spaces, Math. USSR Sb. 57, 527-546.

21. Popov, V.L. (1974) Picard groups of homogeneous spaces of linear algebraic groups and one-dimensional homogeneous vector bundles, Math. USSR Izvestija 8, 301-327.

22. Richardson, R.W. (1977) Affine coset spaces of reductive algebraic groups, Bull. London Math. Soc. 9, 38-41.

23. Richardson, R.W. and Springer, T.A. (1990) The Bruhat order on symmetric varieties, Geom. Ded. 35, 389-436.

24. Richardson, R.W., Röhrle, G. and Steinberg, R. (1992) Parabolic subgroups with abelian unipotent radical, Invent. Math. 110, 649-671.

25. Rosenlicht, M. (1956) Some basic theorems on algebraic groups, Amer. J. Math. 78, 401-443.

26. Sato, M. and Kimura, T. (1977) A classification of irreducible prehomogeneous vector spaces and their relative invariants, Nagoya Math. J. 65, 1-155.

27. Springer, T.A. (1985) Some results on algebraic groups with involutions, in Advanced Studies in Pure Mathematics, vol. 6, North-Holland, pp. 525-543.

28. Vavilov, N. (1997) Weight elements on Chevalley groups, to appear. 
274

29. Vinberg, E.B. (1986) Complexity of action of reductive groups, Funct. Anal. Appl. 20, 1-11. 